\title{
Graphs with constant adjacency dimension
}

\author{
Mohsen Jannesari \\ Faculty of Basic Sciences \\ University of Shahreza \\ 86149-56841, Shahreza, Iran \\ Email:mjannesari@shahreza.ac.ir
}

\begin{abstract}
For a set $W$ of vertices and a vertex $v$ in a graph $G$, the $k$-vector $r_{2}(v \mid W)=\left(a_{G}\left(v, w_{1}\right), \ldots, a_{G}\left(v, w_{k}\right)\right)$ is the adjacency representation of $v$ with respect to $W$, where $W=\left\{w_{1}, \ldots, w_{k}\right\}$ and $a_{G}(x, y)$ is the minimum of 2 and the distance between the vertices $x$ and $y$. The set $W$ is an adjacency resolving set for $G$ if distinct vertices of $G$ have distinct adjacency representations with respect to $W$. The minimum cardinality of an adjacency resolving set for $G$ is its adjacency dimension. It is clear that the adjacency dimension of an $n$-vertex graph $G$ is between 1 and $n-1$. The graphs with adjacency dimension 1 and $n-1$ are known. All graphs with adjacency dimension 2, and all $n$-vertex graphs with adjacency dimension $n-2$ are studied in this paper. In terms of the diameter and order of $G$, a sharp upper bound is found for adjacency dimension of $G$. Also, a sharp lower bound for adjacency dimension of $G$ is obtained in terms of order of $G$. Using these two bounds, all graphs with adjacency dimension 2 , and all $n$-vertex graphs with adjacency dimension $n-2$ are characterized.
\end{abstract}

Keywords: Resolving set; Metric dimension; Metric basis; Adjacency dimension; Diameter.

\section{Introduction}

Throughout this paper, $G$ is a finite simple graph with vertex set $V(G)$, edge set $E(G)$, and order $n(G)$. We use $\bar{G}$ for the complement of $G$. The distance between two vertices $u$ and $v$, denoted by $d_{G}(u, v)$, is the length of a shortest path joining $u$ and $v$ in $G$, we write this simply as $d(u, v)$ when no confusion can arise. The diameter of $G$ is $\operatorname{diam}(G)=\max \{d(u, v) \mid u, v \in V(G)\}$. $N(v)$ is the set of all neighbors of vertex $v$. The edge between adjacent vertices $u$ and $v$, is shown by $u v$. We use $P_{n}$ and $C_{n}$ to denote the isomorphism classes of $n$-vertex paths and cycles, respectively. We use $v_{1}, \ldots, v_{n}$ to denote specific $n$-vertex paths with vertices $v_{1}, \ldots, v_{n}$.

For $W=\left\{w_{1}, \ldots, w_{k}\right\} \subseteq V(G)$ and a vertex $v$ of $G$, the $k$-vector

$$
r(v \mid W)=\left(d\left(v, w_{1}\right), \ldots, d\left(v, w_{k}\right)\right)
$$

is the metric representation of $v$ with respect to $W$. The set $W$ is a resolving set for $G$ if the vertices of $G$ have distinct metric representations, with respect to $W$. A resolving set $W$ for 
$G$ with minimum cardinality is a metric basis of $G$, and its cardinality is the metric dimension of $G$, denoted by $\operatorname{dim}(G)$. The concepts of resolving sets and metric dimension of a graph were introduced independently by Slater [15] and by Harary and Melter [11]. For more results related to these concepts see [1, 2, 3, 5, 7, 14].

Let $G$ and $H$ be two graphs with disjoint vertex sets. The join of $G$ and $H$, denoted by $G \vee H$, is the graph with vertex set $V(G) \cup V(H)$ and edge set $E(G) \cup E(H) \cup\{u v \mid u \in V(G), v \in V(H)\}$. Also, the disjoint union of $G$ and $H$, denoted by $G \cup H$, is the graph with vertex set $V(G) \cup V(H)$ and edge set $E(G) \cup E(H)$. In [6] all graphs of order $n$ with metric dimension $n-2$ are characterized as follows.

Theorem 1.1 [6] Let $G$ be a connected graph of order $n \geq 4$. Then $\operatorname{dim}(G)=n-2$ if and only if $G=K_{s, t}, s, t \geq 1, G=K_{s} \vee \bar{K}_{t}, s \geq 1, t \geq 2$, or $G=K_{s} \vee\left(K_{t} \cup K_{1}\right), s, t \geq 1$.

During the study of the metric dimension of lexicographic product of graphs, Jannesari and Omoomi [13] introduced the concept of adjacency dimension of graphs.

Definition 1.2 [13] Let $G$ be a graph, and $W=\left\{w_{1}, \ldots, w_{k}\right\} \subseteq V(G)$. For each vertex $v \in V(G)$, the adjacency representation of $v$ with respect to $W$ is the $k$-vector

$$
r_{2}(v \mid W)=\left(a_{G}\left(v, w_{1}\right), \ldots, a_{G}\left(v, w_{k}\right)\right),
$$

where

$$
a_{G}\left(v, w_{i}\right)= \begin{cases}0 & \text { if } v=w_{i}, \\ 1 & \text { if } v \text { is adjacent to } w_{i}, \\ 2 & \text { otherwise }\end{cases}
$$

The set $W$ is an adjacency resolving set for $G$ if the vectors $r_{2}(v \mid W)$ for $v \in V(G)$ are distinct. The minimum cardinality of an adjacency resolving set is the adjacency dimension of $G$, denoted by $\operatorname{dim}_{2}(G)$. An adjacency resolving set of cardinality $\operatorname{dim}_{2}(G)$ is an adjacency basis of $G$.

We say that a set $W$ (adjacency) resolves a set $T$ of vertices in $G$, if the adjacency representations of vertices in $T$ with respect to $\mathrm{W}$ are distinct. To determine whether a given set $W$ is an adjacency resolving set for $G$, it is sufficient to look at the adjacency representations of vertices in $V(G) \backslash W$, because $w \in W$ is the unique vertex of $G$ for $\operatorname{which}\left(a_{G}(w, w)=0\right) d(w, w)=0$.

After the introducing of adjacency dimension, researchers interested in studying this parameter, use it for finding metric dimension of some families of graphs and defined some related parameter to adjacency dimension. Fernau and Rodriguez [9, 10] use adjacency dimension to show that the metric dimension of the corona product of a graph of order $n$ and some nontrivial graph $H$ is equal to $n$ times the adjacency dimension of $H$. Using this relationship, they showed that the problem of computing the adjacency dimension is NP-hard. They also define a new related parameter, local adjacency dimension and use it to show that the local metric dimension of the corona product of a graph of order $n$ and some nontrivial graph $H$ is equal to $n$ times the local adjacency dimension of $H$. Estrada et al. [8] introduced the concept of $k$-adjacency dimension and obtained some bounds and closed formulas for some families of graphs. In particular they obtained a closed formula for the $k$-adjacency dimension of join graphs.

By previous works, it is clear that each result about adjacency dimension is important for the study of metric dimension of lexicographic product graphs and corona product graphs. In this 
paper, we find sharp upper and lower bounds for adjacency dimension in terms of diameter and order of a graph. These bounds give us new useful bounds for metric dimension of lexicographic product graphs and corona product graphs.

It is clear that for each graph $G, 1 \leq \operatorname{dim}_{2}(G) \leq n(G)-1$. All graphs with adjacency dimension 1 and all graphs with adjacency dimension $n(G)-1$ are characterized as the following lemma.

Lemma 1.3 [13] Let $G$ be a graph of order $n$.

- $\operatorname{dim}_{2}(G)=n-1$ if and only if $G=K_{n}$ or $G=\bar{K}_{n}$.

- $\operatorname{dim}_{2}(G)=1$ if and only if $G \in\left\{P_{1}, P_{2}, P_{3}, \bar{P}_{2}, \bar{P}_{3}\right\}$.

In Section 2, we find a new sharp upper bound for adjacency dimension in terms of the diameter and order of a graph. Using this bound and characterization of graphs $G$ with metric dimension $n(G)-2$, a characterization of graphs $G$ with adjacency dimension $n(G)-2$ is obtained. In Section 3, a new sharp lower bound for adjacency dimension in terms of the order of a graph is presented. All graphs that attain this bound are characterized. This bound and some known results lead us to a characterization of graphs with adjacency dimension 2 .

The next results about adjacency dimension of graphs is needed in the following.

Lemma 1.4 [13] Let $G$ be a graph of order $n$.

- If $\operatorname{diam}(G)=2$, then $\operatorname{dim}_{2}(G)=\operatorname{dim}(G)$.

- If $G$ is connected, then $\operatorname{dim}_{2}(G) \geq \operatorname{dim}(G)$.

- $\operatorname{dim}_{2}(G)=\operatorname{dim}_{2}(\bar{G})$.

- If $n \geq 4$, then $\operatorname{dim}_{2}\left(C_{n}\right)=\operatorname{dim}_{2}\left(P_{n}\right)=\left\lfloor\frac{2 n+2}{5}\right\rfloor$.

Two distinct vertices $u$ and $v$ are twins if $N(v) \backslash\{u\}=N(u) \backslash\{v\}$. It is easy to see that, if $u, v$ are twins in $G$ then for each $x \in V(G) \backslash\{u, v\}, d(x, u)=d(x, v)$ and therefore $a_{G}(x, u)=a_{G}(x, v)$. Thus, we have the following lemma.

Lemma 1.5 If $u, v$ are twin vertices in a graph $G$, then every adjacency resolving set for $G$ contains at least one of the vertices $u$ and $v$.

\section{Graphs of order $n$ and adjacency dimension $n-2$}

This section is aimed to characterize all $n$-vertex graphs with adjacency dimension $n-2$. To gain this goal, we first find a sharp upper bound for adjacency dimension of graphs in terms of its order and diameter.

Lemma 2.1 Let $G$ be a connected graph of order $n$ and diameter $D$. Then

$$
\operatorname{dim}_{2}(G) \leq n-D-1+\left\lfloor\frac{2 D+4}{5}\right\rfloor
$$


Proof. If $D=1$, then $G=K_{n}$ and $\operatorname{dim}_{2}(G)=n-1=n-D-1+\left\lfloor\frac{2 D+4}{5}\right\rfloor$. Now we consider $D \geq 2$. Let $u, v \in V(G)$ be two vertices with $d(u, v)=D$ and $P_{D+1}=u, u_{2}, u_{3}, \ldots, u_{D}, v$ be a shortest path between $u$ and $v$. If $D=2$, then $\operatorname{dim}_{2}\left(P_{3}\right)=1=\left\lfloor\frac{2 D+4}{5}\right\rfloor$. For $D \geq 3$, by Lemma 1.4, $\operatorname{dim}_{2}\left(P_{D+1}\right)=\left\lfloor\frac{2 D+4}{5}\right\rfloor=t$. Let $B=\left\{v_{1}, v_{2}, \ldots, v_{t}\right\} \subseteq\left\{u, u_{2}, u_{3}, \ldots, u_{D}, v\right\}$ be an adjacency basis of $P_{D+1}$. Consider the set $W=\left(V(G)-V\left(P_{D+1}\right)\right) \cup B$. If there exist vertices $x, y \in V(G)-W \subseteq V\left(P_{D+1}\right)$ with $r_{2}(x \mid W)=r_{2}(y \mid W)$, then $r_{2}(x \mid B)=r_{2}(y \mid B)$ and this is a contradiction, because $B$ is an adjacency basis of $P_{D+1}$. Thus, $W$ is an adjacency resolving set for $G$ with cardinality $n-D-1+\left\lfloor\frac{2 D+4}{5}\right\rfloor$.

It is clear that upper bound in Theorem 2.1 is tight for $G=P_{n}$ and $G=K_{n}$. In the next theorem, we construct an infinite family of graphs of diameter $D$, order $n \geq D+1$ and adjacency dimension $n-D-1+\left\lfloor\frac{2 D+4}{5}\right\rfloor$. Therefore upper bound in Lemma 2.1 is sharp.

Theorem 2.2 Let $k$ be a positive integer and $D \in\{5 k, 5 k+2\}$. Then for each integer $n \geq D+1$ there exists a graph $G$ with $n$ vertices and diameter $D$, such that $\operatorname{dim}_{2}(G)=n-D-1+\left\lfloor\frac{2 D+4}{5}\right\rfloor$.

Proof. Let $G$ be a graph with $V(G)=\left\{v_{0}, v_{1}, v_{2}, \ldots, v_{D}\right\} \cup\left\{u_{1}, u_{2}, \ldots, u_{n-D-1}\right\}$ and $E(G)=$ $\left\{v_{i} v_{i+1} \mid 0 \leq i \leq D-1\right\} \cup\left\{v_{i} u_{j} \mid 0 \leq i \leq 2,1 \leq j \leq n-D-1\right\} \cup\left\{u_{i} u_{j} \mid 1 \leq i, j \leq n-D-1\right\}$, see Figure ??. Clearly $G$ has $n$ vertices and $\operatorname{diam}(G)=D$. We prove that $\operatorname{dim}_{2}(G)=$ $n-D-1+\left\lfloor\frac{2 D+4}{5}\right\rfloor$.

If $n=D+1$, then there is no any vertex $u_{i}$ in $G$ and $G=P_{D+1}$. Thus, by Lemma 1.4 $\operatorname{dim}_{2}(G)=\left\lfloor\frac{2(D+1)+2}{5}\right\rfloor=n-D-1+\left\lfloor\frac{2 D+4}{5}\right\rfloor$.

Now let $n \geq D+2$ and $B$ be an adjacency basis of $G$. In this case, the set $U=$ $\left\{v_{1}, u_{1}, u_{2}, \ldots, u_{n-D-1}\right\}$ is a set of twin vertices. So, by Lemma 1.5, at most one of the vertices of $U$ can be not in $B$, say $U-B \subseteq\{u\}$. Note that, $r_{2}(u \mid U-\{u\})=r_{2}\left(v_{0} \mid U-\{u\}\right)=$ $r_{2}\left(v_{2} \mid U-\{u\}\right)=(1,1, \ldots, 1)$ and $r_{2}\left(v_{i} \mid U-\{u\}\right)=(2,2, \ldots, 2)$, for $i \geq 3$. Since none of vertices $v_{3}, v_{4}, \ldots, v_{D}$ can adjacency resolve vertices $v_{0}$ and $v_{1}$, to adjacency resolve $\left\{u, v_{0}, v_{2}\right\}$, we need at least one vertex from this set, say $y$. But, $r_{2}\left(v_{i} \mid(U \cup\{y\})-\{u\}\right)=(2,2, \ldots, 2)$, for $i \geq 4$. Since $v_{i}$ 's, $4 \leq i \leq D$, form a path of order $D-3$, by Lemma 1.4 to adjacency resolve these vertices we need $\left\lfloor\frac{2(D-3)+2}{5}\right\rfloor$ vertices from this set. Therefore

$\operatorname{dim}_{2}(G) \geq|(U \cup\{y\})-\{u\}|+\left\lfloor\frac{2(D-3)+2}{5}\right\rfloor=n-D+\left\lfloor\frac{2(D-3)+2}{5}\right\rfloor=n-D+\left\lfloor\frac{2 D-4}{5}\right\rfloor$.

On the other hand, if $B_{1}$ is a basis of $P_{D-3}=v_{4}, v_{5}, \ldots, v_{D}$, then the set $\left\{v_{2}, u_{1}, u_{2}, \ldots, u_{n-D-1}\right\} \cup$ $B_{1}$ is an adjacency resolving set for $G$ of size $n-D+\left\lfloor\frac{2 D-4}{5}\right\rfloor$. Therefore, $\operatorname{dim}_{2}(G)=n-D+$ $\left\lfloor\frac{2 D-4}{5}\right\rfloor$.

In case $D=5 k$, we have

$$
\left\lfloor\frac{2 D-4}{5}\right\rfloor=\left\lfloor\frac{10 k-4}{5}\right\rfloor=2 k-1=\left\lfloor\frac{10 k+4}{5}\right\rfloor-1=\left\lfloor\frac{2 D+4}{5}\right\rfloor-1 .
$$

And in case $D=5 k+2$,

$$
\left\lfloor\frac{2 D-4}{5}\right\rfloor=\left\lfloor\frac{10 k+4-4}{5}\right\rfloor=2 k=\left\lfloor\frac{10 k+4+4}{5}\right\rfloor-1=\left\lfloor\frac{2 D+4}{5}\right\rfloor-1 .
$$

Therefore in these two cases, $\operatorname{dim}_{2}(G)=n-D-1+\left\lfloor\frac{2 D+4}{5}\right\rfloor$. 
For arbitrary $D$ and each $n \geq D+1$ the following question is propounded.

Question 2.3 Is there a graph $G$ of diameter $D$ with $\operatorname{dim}_{2}(G)=n-D-1+\left\lfloor\frac{2 D+4}{5}\right\rfloor$, for each $D$ and $n \geq D+1$.

All graphs of order $n$ with metric dimension $n-2$ are characterized in Theorem 1.1. Through the next theorem all graphs of order $n$ and adjacency dimension $n-2$ are characterised.

Theorem 2.4 Let $G$ be a graph of order $n$. Then $\operatorname{dim}_{2}(G)=n-2$ if and only if $G$ or $\bar{G}$ is one of the graphs $P_{4}, K_{s, t}(s, t \geq 1), K_{s} \vee \bar{K}_{t}(s \geq 1, t \geq 2)$, or $K_{s} \vee\left(K_{t} \cup K_{1}\right)(s, t \geq 1)$.

Proof. If $G$ or $\bar{G}$ is one of the graphs $P_{4}, K_{s, t}(s, t \geq 1), K_{s} \vee \bar{K}_{t}(s \geq 1, t \geq 2)$, or $K_{s} \vee\left(K_{t} \cup\right.$ $\left.K_{1}\right)(s, t \geq 1)$, then it is clear that $\operatorname{dim}_{2}(G)=n-2$.

Conversely, we first prove for connected graphs. Let $G$ be a connected graph of order $n$ and $\operatorname{dim}_{2}(G)=n-2$. If $\operatorname{diam}(G) \geq 4$, then by Lemma 2.1,

$$
n-2 \leq n-4-1+\left\lfloor\frac{8+4}{5}\right\rfloor=n-3 .
$$

This contradiction implies $\operatorname{diam}(G) \leq 3$. If $\operatorname{diam}(G) \leq 2$, then by $\operatorname{Lemma}_{1.4} \operatorname{dim}_{2}(G)=$ $\operatorname{dim}(G)$, thus by Theorem $2 G$ is one of the graphs, $K_{s, t}(s, t \geq 1), K_{s} \vee \bar{K}_{t}(s \geq 1, t \geq 2)$, or $K_{s} \vee\left(K_{t} \cup K_{1}\right)(s, t \geq 1)$.

Now let $\operatorname{diam}(G)=3$ and $x, y \in V(G)$ such that $d(x, y)=3$. Suppose that $N_{i}(x)=\{t \in$ $V(G) \mid d(x, t)=i\}, 0 \leq i \leq 3$. If there exist non-adjacent vertices $b \in N_{2}(x)$ and $c \in N_{3}(x)$, then $c$ has a neighbour $b^{\prime} \neq b$ in $N_{2}(x)$ and

$$
r_{2}(b \mid\{x, c\})=(2,2), \quad r_{2}\left(b^{\prime} \mid\{x, c\}\right)=(2,1), \quad r_{2}(e \mid\{x, c\})=(1,2),
$$

where $e$ is an arbitrary vertex in $N_{1}(x)$. Since these three adjacency representations are distinct, $V(G)-\left\{b, b^{\prime}, e\right\}$ is an adjacency resolving set for $G$. This contradiction implies that all vertices of $N_{3}(x)$ are adjacent to all vertices in $N_{2}(x)$. If there exist non-adjacent vertices $b \in N_{1}(x)$ and $c \in N_{2}(x)$, then $c$ has a neighbour $b^{\prime} \neq b$ in $N_{1}(x)$ and

$$
r_{2}(b \mid\{x, c\})=(1,2), \quad r_{2}\left(b^{\prime} \mid\{x, c\}\right)=(1,1), \quad r_{2}(e \mid\{x, c\})=(2,1),
$$

where $e$ is an arbitrary vertex in $N_{3}(x)$. Hence $V(G)-\left\{b, b^{\prime}, e\right\}$ is an adjacency resolving set for $G$. This contradiction implies that all vertices of $N_{2}(x)$ are adjacent to all vertices in $N_{1}(x)$. If $u, v \in N_{1}(x)$ are two distinct vertices, then

$$
r_{2}(y \mid\{x, u\})=(2,2), \quad r_{2}(z \mid\{x, u\})=(2,1), \quad r_{2}(v \mid\{x, u\})=(1, i),
$$

where $z$ is an arbitrary vertex in $N_{2}(x)$ and $i \in\{1,2\}$. Hence $V(G)-\{y, z, v\}$ is an adjacency resolving set for $G$. This contradiction implies that $\left|N_{1}(x)\right|=1$, say $N_{1}(x)=\{w\}$. If $u, v \in$ $N_{2}(x)$ are two distinct vertices, then

$$
r_{2}(x \mid\{y, u\})=(2,2), \quad r_{2}(w \mid\{y, u\})=(2,1), \quad r_{2}(v \mid\{y, u\})=(1, i),
$$

where $i \in\{1,2\}$. Hence $V(G)-\{x, w, v\}$ is an adjacency resolving set for $G$. This contradiction implies that $\left|N_{2}(x)\right|=1$, say $N_{2}(x)=\{z\}$. If $u, v \in N_{3}(x)$ are two distinct vertices, then

$$
r_{2}(x \mid\{w, u\})=(1,2), \quad r_{2}(z \mid\{w, u\})=(1,1), \quad r_{2}(v \mid\{w, u\})=(2, i),
$$


where $i \in\{1,2\}$. Hence $V(G)-\{x, z, v\}$ is an adjacency resolving set for $G$. This contradiction implies that $\left|N_{3}(x)\right|=1$. Therefore $G=P_{4}$.

Since the complement of a disconnected graph is connected, $G$ or $\bar{G}$ is one of the graphs $P_{4}$, $K_{s, t}(s, t \geq 1), K_{s} \vee \bar{K}_{t}(s \geq 1, t \geq 2)$, or $K_{s} \vee\left(K_{t} \cup K_{1}\right)(s, t \geq 1)$.

\section{Graphs with adjacency dimension 2}

In this Section, a sharp upper bound for order of graphs with adjacency dimension $k$ is presented. This leads us to a lower bound for adjacency dimension of graphs in terms of order of a graph. In fact if we consider $f(n)$ as the least positive integer $k$ such that $k+2^{k} \geq n(G)$, then for each graph $G$ of order $n$, $\operatorname{dim}_{2}(G) \leq f(n)$. All graphs that attain this bound are characterized. This bound and some known results lead us to a characterization of graphs with adjacency dimension 2 .

Lemma 3.1 Let $G$ be a graph of order $n$ and $\operatorname{dim}_{2}(G)=k$. Then $n \leq k+2^{k}$.

Proof. Let $B$ be an adjacency basis of $G$. For each vertex $v \in V(G), r_{2}(v \mid B)$ is a $k$-vector with entries $0,1,2$. The members of $B$ are all vertices that their representation have entry 0 . The representation of other vertices are constructed by 1 and 2 . Therefore, $G \backslash B$ has at most $2^{k}$ vertices. That is $n \leq k+2^{k}$.

In the next example we construct a family of graphs with $6=2+2^{2}$ vertices and adjacency dimension 2 .

Example 3.2 Let $n(G)=6$, we introduce a construction for graphs with adjacency dimension 2. Let $H$ and $K$ be two arbitrary graphs with $V(H)=\{a, b\}$ and $V(K)=\{c, d, e, f\}$. Consider graph $G$ with $V(G)=\{a, b, c, d, e, f\}$ and $E(G)=E(H) \cup E(K) \cup\{a c, a d, b d, b e\}$. By Lemma 3.1, $\operatorname{dim}_{2}(G) \geq 2$. On the other hand,

$$
r_{2}(c \mid\{a, b\})=(1,2), \quad r_{2}(d \mid\{a, b\})=(1,1), \quad r_{2}(e \mid\{a, b\})=(2,1), \quad r_{2}(f \mid\{a, b\})=(2,2) .
$$

Hence, $\{a, b\}$ is an adjacency resolving set for $G$ of size 2 . Therefore $\operatorname{dim}_{2}(G)=2$.

By extending the construction in Example 3.2, we construct an infinite family of graphs with adjacency dimension $k$ and order $k+2^{k}$ in the next theorem. In fact in this theorem for each positive integer $k$, we find all graphs with adjacency dimension $k$ and order $k+2^{k}$.

Theorem 3.3 For each positive integer $k$, the family $\Omega_{k}$ of all graphs with adjacency dimension $k$ and order $k+2^{k}$ has $2^{\left(\begin{array}{c}k \\ 2\end{array}\right)+\left(\begin{array}{c}2^{k} \\ 2\end{array}\right)}$ members.

Proof. Let $k$ be a positive integer and $G$ be a graph with vertex set $V(G)=\left\{v_{1}, v_{2}, \ldots, v_{k}\right\} \cup$ $\left\{u_{1}, u_{2}, \ldots, u_{2^{k}}\right\}$, where $u_{i}$ 's are $k$-vectors with entries 1 and 2 . A vertex $v_{i}$ is adjacent to $u_{j}$ if the $i$ th entry of $u_{j}$ is 1 . The adjacency of two members of $V=\left\{v_{1}, v_{2}, \ldots, v_{k}\right\}$ is arbitrary. Also the adjacency of two members of $U=\left\{u_{1}, u_{2}, \ldots, u_{2^{k}}\right\}$ is arbitrary. We define the family $\Omega_{k}$ all these graphs. Since the adjacency of two members of $V$ and two members of $U$ are arbitrary, 
$\left|\Omega_{k}\right|=2^{\left(\begin{array}{c}k \\ 2\end{array}\right)+\left(\begin{array}{c}2^{k} \\ 2\end{array}\right)}$. Now we prove that the family $\Omega_{k}$ consists of all graphs with adjacency dimension $k$ and order $k+2^{k}$.

Let $G$ be a graph in $\Omega_{k}$, by Lemma 3.1, $\operatorname{dim}_{2}(G) \geq k$. On the other hand, for each $u_{i}$, $1 \leq i \leq 2^{k}$, the adjacency representation of $u_{i}$ with respect to $V$ is its corresponding $k$-vector, that is the adjacency representation of all vertices of $U$ with respect to $V$ are deferent. Also for each $i, 1 \leq i \leq k, v_{i}$ is the unique vertex of $G$ with 0 in $i$ th entry in $r_{2}\left(v_{i} \mid V\right)$, thus $V$ is an adjacency resolving set for $G$ of size $k$. Therefore the adjacency dimension of all members of $\Omega_{k}$ is $k$.

Now we need to prove that each graph with adjacency dimension $k$ and order $k+2^{k}$ belongs to $\Omega_{k}$. Let $G$ be a graph with this properties, $V$ be its adjacency basis and $U=V(G) \backslash V$. Hence $|V|=k$ and there are $2^{k}$ distinct adjacency representations with respect to $V$ for vertices in $U$. Since $|U|=2^{k}$, the set of adjacency representations of vertices in $U$ with respect to $V$ is the set of all $k$-vectors with entries 1 and 2 . That is, $U$ is correspond to the set of all $k$-vectors with entries 1 and 2. Also a vertex $v_{i} \in V$ is adjacent to a vertex $u_{j} \in U$ if and only if the $i$ th entry of its adjacency representation is 1 . Therefore $G \in \Omega_{k}$.

In the remaining of this section we find all graphs with adjacency dimension 2. By Lemma 3.1, if $\operatorname{dim}_{2}(G)=2$, then $n(G) \leq 6$. Thus to study graphs with adjacency dimension 2 , it is sufficient to consider all graphs with at most 6 vertices. In the next, all graphs $G$ with order at most 6 are studied and in each case all graphs with adjacency dimension 2 are detected.

Case $1 . n(G) \leq 2$ : in this case, all graphs has adjacency dimension 1 .

Case 2. $n(G)=3$, in this case, $n-1=2$ and by Lemma $1.3, \operatorname{dim}_{2}(G)=2$ if and only if $G=K_{3}$ or $G=\bar{K}_{3}$.

Case 3. $n(G)=4$, in this case by Lemma 3.1, $\operatorname{dim}_{2}(G) \geq 2$. Clearly $\operatorname{dim}_{2}(G) \leq n(G)-1=3$. But $\operatorname{dim}_{2}(G)=3$ if and only if $G=K_{4}$ or $G=\bar{K}_{4}$. Therefore $\operatorname{dim}_{2}(G)=2$ if and only if $G$ is not $K_{4}$ or $\bar{K}_{4}$.

Case 4. $n(G)=5$, Lemma 3.1 implies that $\operatorname{dim}_{2}(G) \geq 2$, clearly $\operatorname{dim}_{2}(G) \leq n(G)-1=4$. Also, by Lemma $1.3 \operatorname{dim}_{2}(G)=4$ if and only if $G=K_{5}$ or $G=\bar{K}_{5}$. Moreover, Theorem 2.4 implies $\operatorname{dim}_{2}(G)=3$ if and only if $G$ or $\bar{G}$ is one of the graphs, $K_{1,4}, K_{2,3}, K_{3} \vee \bar{K}_{2}, K_{2} \vee \bar{K}_{3}, K_{1} \vee$ $\left(K_{3} \cup K_{1}\right)$ or $K_{2} \vee\left(K_{2} \cup K_{1}\right)$. Therefore, $\operatorname{dim}_{2}(G)=2$ if and only if $G$ or $\bar{G}$ is not any of the following graphs.

$$
K_{5}, K_{1,4}, K_{2,3}, K_{3} \vee \bar{K}_{2}, K_{2} \vee \bar{K}_{3}, K_{1} \vee\left(K_{3} \cup K_{1}\right), K_{2} \vee\left(K_{2} \cup K_{1}\right)
$$

Case 5. $n(G)=6$, in this case the family of all graphs is $\Omega_{2}$. In fact these graphs are described in Example 3.2 .

Therefore we found all graphs with adjacency dimension 2.

\section{References}

[1] R.F. Bailey and P.J. Cameron, Base size, metric dimension and other invariants of groups and graphs, Bull. London Math. Soc. 43 (2011) 209-242. 
[2] R.C. Brigham, G. Chartrand, R.D. Dutton, and P. Zhang, On the dimension of trees, Discrete Mathematics 294 (2005) 279-283.

[3] P.S. Buczkowski, G. Chartrand, C. Poisson, and P. Zhang, On k-dimensional graphs and their bases, Periodica Mathematica Hungarica 46(1) (2003) 9-15.

[4] J. Caceres, C. Hernando, M. Mora, I.M. Pelayo, M.L. Puertas, C. Seara, and D.R. Wood, On the metric dimension of cartesian products of graphs, SIAM Journal Discrete Mathematics 21(2) (2007) 423-441.

[5] G.G. Chappell, J. Gimbel, and C. Hartman, Bounds on the metric and partition dimensions of a graph, Ars Combinatoria 88 (2008) 349-366.

[6] G. Chartrand, L. Eroh, M.A. Johnson, and O.R. Ollermann, Resolvability in graphs and the metric dimension of a graph, Discrete Applied Mathematics 105 (2000) 99-113.

[7] G. Chartrand and P. Zhang, The theory and applications of resolvability in graphs. A survey. In Proc. 34-th Southeastern International Conf. on Combinatorics, Graph Theory and Computing 160 (2003) 47-68.

[8] A. Estrada-Moreno, Y. Ramirez-Cruz, and J.A. Rodriguez-Velazquez, On the adjacency dimension of graphs. Applicable Analysis and Discrete Mathematics 10 (2016) 102-127.

[9] H. Fernau and J.A. Rodriguez-Velazquez, On the (adjacency) metric dimension of corona and strong product graphs and their local variants: Combinatorial and computational results. Discrete Applied Mathematics 236 (2018) 183-202.

[10] H. Fernau and J.A. Rodriguez-Velazquez, Notions of metric dimension of corona products: Combinatorial and computational results. Lecture Notes in Comput. Sci. 8476 (2014) 153166 .

[11] F. Harary and R.A. Melter, On the metric dimension of a graph, Ars Combinatoria 2 (1976) 191-195.

[12] C. Hernando, M. Mora, I.M. Pelayo, C. Seara, and D.R. Wood, Extremal Graph Theory for Metric Dimension and Diameter, The Electronic Journal of Combinatorics 17 (2010) \#R30.

[13] M. Jannesari and B. Omoomi, The metric dimension of the lexicographic product of graphs, Discrete Mathematics. 312(22) (2012) 3349-3356.

[14] S. Khuller, B. Raghavachari, and A. Rosenfeld, Landmarks in graphs, Discrete Applied Mathematics 70(3) (1996) 217-229.

[15] P.J. Slater, Leaves of trees, Congressus Numerantium 14 (1975) 549-559. 